Article

\title{
Self-Adjointness and Conservation Laws of Frobenius Type Equations
}

\author{
Haifeng Wang and Yufeng Zhang * \\ School of Mathematics, China University of Mining and Technology, Xuzhou 221116, China; \\ tb19080005b4@cumt.edu.cn \\ * Correspondence: zyfxz@cumt.edu.cn
}

Received: 14 November 2020; Accepted: 30 November 2020; Published: 1 December 2020

\begin{abstract}
The Frobenius KDV equation and the Frobenius KP equation are introduced, and the Frobenius Kompaneets equation, Frobenius Burgers equation and Frobenius Harry Dym equation are constructed by taking values in a commutative subalgebra $\mathcal{Z}_{2}^{\varepsilon}$ in the paper. The five equations are selected as examples to help us study the self-adjointness of Frobenius type equations, and we show that the first two equations are quasi self-adjoint and the last three equations are nonlinear self-adjointness. It follows that we give the symmetries of the Frobenius KDV and the Frobenius KP equation in order to construct the corresponding conservation laws.
\end{abstract}

Keywords: quasi self-adjointness to Frobenius type equations; nonlinear self-adjointness to Frobenius type equations; symmetry; conservation laws

PACS: 05.45.Yv; 02.30.Rz; 02.30.Ik; 45.20.Jj; 02.90.Qs

\section{Introduction}

In the field of mathematical and physical science, nonlinear partial differential equations (NLPDE) have wide applications, including nonlinear optics [1], fluid flows [2], plasma physics [3], excitable media [4], and so on [5,6]. The investigation of conservation law plays a significant role in analyzing the (NLPDE). Some fields involve conservation laws, such as jet flows [7], inverse scattering transformation, reductions of the equations [8], Lax operators, and exact solutions [9]. There are numerous effective methods for deducing the conservation laws, such as the Noether approach, the direct method, the variational approach and the nonlinear self-adjointness. The connection between symmetries of differential equations and conservation laws was established by Noether's theorem [10]. N.H. Ibragimov proposed the method of nonlinear self-adjointness in [11]. It follows that the general theorem on conservation laws for arbitrary differential equations was given by [12], and successively developed in $[13,14]$. Based on the method, the conservation laws of some NLPDE models were obtained [15-18], which can be used to study the properties of the NLPDE models in further study.

In [19], the authors construct the Frobenius-valued Kakomtsev-Petviashvili hierarchy, and then Frobenius KDV equation, Frobenius Camassa-Holm equation, Frobenius Hunter-Saxton equation, and so on were obtained based on the Frobenius-Virasoro algebra in [20]. It follows that Li considered the Hirota quadratic equation of the commutative version of extended multi-component Toda hierarchy in [21], and the gauge transformation and symmetries of the $\mathcal{Z}_{n}$-BKP hierarchy were investigated in [22]. Moreover, we studied the Bäcklund transformation and affine Weyl group symmetries of Frobenius Painlevé equations of type $\mathcal{Z}_{2}^{\varepsilon}$ and $\mathcal{Z}_{n}$ [23,24], respectively. Additionally, the residual Symmetries of the Strongly Coupled Boussinesq-Burgers System was also studied by us in [25]. 
The related research on Frobenius equations has become an important subject of mathematical physics, such as, conservation laws, symmetry, soliton solution, and so on. It is known that once the symmetry and conservation laws of an equation are obtained, the solution of this equation can be found, such as rough solution, lump solution and soliton solution, and so on. A natural problem then arises as to how to construct conservation laws for these Frobenius equations. Therefore, our systematic study of the conservation law of the Frobenius equation has made substantial preparations for the next systematic study of the solution of the Frobenius equation. The goal of this paper is to investigate the self-adjointness and conservation laws of Frobenius type equations by picking some Frobenius equations as examples. In order to study the quasi self-adjointness of the Frobenius type equation, we introduce the Frobenius KDV equation and Frobenius KP equation. It follows that the Frobenius Kompaneets equation, Frobenius Burgers equation, and Frobenius Harry Dym equation are constructed for investigating the nonlinear self-adjointness of the Frobenius type equation. We choose these five Frobenius equations because the corresponding single-component equations, incuding the KP equation, the KDV equation, the Kompaneets equation, the Burgers equation and the Harry Dym equation are very representative in mathematical physics and related fields. After that the conservation laws of Frobenius type equations are found by means of symmetries.

\section{Quasi Self-Adjointness of Frobenius Type Equations}

To investigate the self-adjointness of Frobenius type equations, we first introduce the notation as follows:

$$
\begin{aligned}
& x=\left(x^{1}, \ldots, x^{n}\right), \\
& u_{i}^{\alpha}=D_{i}\left(u^{\alpha}\right), u_{i j}^{\alpha}=D_{i} D_{j}\left(u^{\alpha}\right), \ldots, v_{i}^{\alpha}=D_{i}\left(v^{\alpha}\right), v_{i j}^{\alpha}=D_{i} D_{j}\left(v^{\alpha}\right), \ldots, \\
& \bar{u}_{i}^{\alpha}=D_{i}\left(\bar{u}^{\alpha}\right), \bar{u}_{i j}^{\alpha}=D_{i} D_{j}\left(\bar{u}^{\alpha}\right), \ldots, \quad \bar{v}_{i}^{\alpha}=D_{i}\left(\bar{v}^{\alpha}\right), \bar{v}_{i j}^{\alpha}=D_{i} D_{j}\left(\bar{v}^{\alpha}\right), \ldots,
\end{aligned}
$$

where $x^{k}(k=1,2, \ldots, n)$ are independent variables and $D_{i}$ denote the total differentiations operator with four dependent variables $u, v, \bar{u}, \bar{v}$, i.e.,

$$
D_{i}=\frac{\partial}{\partial x^{i}}+u_{i}^{\alpha} \frac{\partial}{\partial u^{\alpha}}+v_{i}^{\alpha} \frac{\partial}{\partial v^{\alpha}}+\bar{u}_{i}^{\alpha} \frac{\partial}{\partial \bar{u}^{\alpha}}+\bar{v}_{i}^{\alpha} \frac{\partial}{\partial \bar{v}^{\alpha}}+u_{i j}^{\alpha} \frac{\partial}{\partial u_{j}^{\alpha}}+v_{i j}^{\alpha} \frac{\partial}{\partial v_{j}^{\alpha}}+\bar{u}_{i j}^{\alpha} \frac{\partial}{\partial \bar{u}_{j}^{\alpha}}+\bar{v}_{i j}^{\alpha} \frac{\partial}{\partial \bar{v}_{j}^{\alpha}}+\cdots .
$$

The Frobenius equations of $m$ differential equations can be written as

$$
F_{\alpha}\left(x, u, \bar{u}, u_{(1)}, \bar{u}_{(1)}, \ldots, u_{(s)}, \bar{u}_{(s)}\right)=0, \alpha=1, \ldots, m,
$$

which admits the adjoint equations (see $[13,14])$

$$
F_{\alpha}^{*}\left(x, u, v, \bar{u}, \bar{v}, u_{(1)}, v_{(1)}, \bar{u}_{(1)}, \bar{v}_{(1)}, \ldots, u_{(s)}, v_{(s)}, \bar{u}_{(s)}, \bar{v}_{(s)}\right)=0, \alpha=1, \ldots, m,
$$

where

$$
\begin{aligned}
& F_{\alpha}^{*}\left(x, u, v, \bar{u}, \bar{v}_{1} u_{(1)}, v_{(1)}, \bar{u}_{(1)}, \bar{v}_{(1)}, \ldots, u_{(s)}, v_{(s)}, \bar{u}_{(s)}, \bar{v}_{(s)}\right)=\frac{\delta \mathcal{L}}{\delta u^{\alpha}}, \\
& u_{(1)}=\left\{u_{i}^{\alpha}\right\}, u_{(2)}=\left\{u_{i j}^{\alpha}\right\}, \cdots, u_{(s)}=\left\{u_{i_{1} \cdots i_{s}}^{\alpha}\right\}, v_{(1)}=\left\{v_{i}^{\alpha}\right\}, v_{(2)}=\left\{v_{i j}^{\alpha}\right\}, \cdots, v_{(s)}=\left\{v_{i_{1} \cdots i_{s}}^{\alpha}\right\}, \\
& \bar{u}_{(1)}=\left\{\bar{u}_{i}^{\alpha}\right\}, \bar{u}_{(2)}=\left\{\bar{u}_{i j}^{\alpha}\right\}, \cdots, \bar{u}_{(s)}=\left\{\bar{u}_{i_{1} \cdots i_{s}}^{\alpha}\right\}, \bar{v}_{(1)}=\left\{\bar{v}_{i}^{\alpha}\right\}, \bar{v}_{(2)}=\left\{\bar{v}_{i j}^{\alpha}\right\}, \cdots, \bar{v}_{(s)}=\left\{\bar{v}_{i_{1} \cdots i_{s}}^{\alpha}\right\},
\end{aligned}
$$

with $\mathcal{L}$ is the formal Lagrangian for Equation (1) given by

$$
\mathcal{L}=\sum_{\alpha=1}^{m} v^{\alpha} F_{\alpha}\left(x, u, \bar{u}, u_{(1)}, \bar{u}_{(1)}, \ldots, u_{(s)}, \bar{u}_{(s)}\right)+\sum_{\beta=1}^{m} \bar{v}^{\beta} F_{\beta}\left(x, u, \bar{u}, u_{(1)}, \bar{u}_{(1)}, \ldots, u_{(s)}, \bar{u}_{(s)}\right),
$$


and $\frac{\delta}{\delta u^{\alpha}}$ is the variational derivative reads that

$$
\frac{\delta}{\delta u^{\alpha}}=\frac{\partial}{\partial u^{\alpha}}+\sum_{s=1}^{\infty}(-1)^{s} D_{i_{1}} \cdots D_{i_{s}} \frac{\partial}{\partial u_{i_{1} \cdots i_{s}}^{\alpha}} .
$$

Definition 1. If the adjoint Equation (2) becomes equivalent to the original system (1) upon the substitution $($ see $[13,14])$

$$
v^{\alpha}=u^{\alpha}, \bar{v}^{\alpha}=\bar{u}^{\alpha}, \alpha=1, \ldots, m,
$$

then we call the Frobenius system (1) is strictly self-adjoint.

Definition 2. If the adjoint Equation (2) is satisfied for all solutions $u$ and $\bar{u}$ of the system (1) upon a substitution (see $[13,15])$

$$
v^{\alpha}=\varphi^{\alpha}(u), \bar{v}^{\alpha}=\bar{\varphi}^{\alpha}(\bar{u}), \alpha=1, \ldots, m,
$$

such that

$$
\varphi^{\alpha}(u) \neq 0, \bar{\varphi}^{\alpha}(\bar{u}) \neq 0,
$$

then we call the Frobenius system (1) is quasi self-adjoint.

That is to say the following equations hold with undetermined coefficients $\lambda_{\alpha}^{\beta}$ :

$$
F_{\alpha}^{*}\left(x, u, \varphi, \bar{u}, \bar{\varphi}, u_{(1)}, \varphi_{(1)}, \bar{u}_{(1)}, \bar{\varphi}_{(1)}, \ldots, u_{(s)}, \varphi_{(s)}, \bar{u}_{(s)}, \bar{\varphi}_{(s)}\right)=\lambda_{\alpha}^{\beta} F_{\beta}\left(x, u, \bar{u}, u_{(1)}, \bar{u}_{(1)}, \ldots, u_{(s)}, \bar{u}_{(s)}\right),
$$

where

$$
\varphi=\left\{\varphi^{\alpha}(u)\right\}, \bar{\varphi}=\left\{\bar{\varphi}^{\alpha}(\bar{u})\right\}, \varphi_{k}=\left\{D_{i} \ldots D_{k}\left(\varphi^{\alpha}(u)\right)\right\}, \bar{\varphi}_{k}=\left\{D_{i} \ldots D_{k}\left(\bar{\varphi}^{\alpha}(\bar{u})\right)\right\}, k=1, \ldots, s,
$$

and $\varphi^{\alpha}(u) \neq 0$ means that not all components $\varphi^{\alpha}(u)$ of the $\varphi$ vanish simultaneously, so does $\bar{\varphi}^{\alpha}(\bar{u})$.

Example 1. Let us consider the Frobenius KDV equation (see [20])

$$
\left\{\begin{array}{l}
u_{t}=u_{x x x}+u u_{x}+\varepsilon \overline{u u}_{x} \\
\bar{u}_{t}=\bar{u}_{x x x}+u \bar{u}_{x}+\bar{u} u_{x}
\end{array}\right.
$$

which can be written as

$$
\left\{\begin{array}{l}
F_{1}=-u_{t}+u_{x x x}+u u_{x}+\varepsilon \overline{u u}_{x}=0, \\
F_{2}=-\bar{u}_{t}+\bar{u}_{x x x}+u \bar{u}_{x}+\bar{u} u_{x}=0 .
\end{array}\right.
$$

By using the above notations and formulas, the formal Lagrangian of the Frobenius KDV Equation (7) reads

$$
\mathcal{L}=v\left(-u_{t}+u_{x x x}+u u_{x}+\varepsilon \bar{u}_{x}\right)+\bar{v}\left(-\bar{u}_{t}+\bar{u}_{x x x}+u \bar{u}_{x}+\bar{u} u_{x}\right)
$$

admits the adjoint system

$$
\left\{\begin{array}{l}
F_{1}^{*}=\frac{\delta \mathcal{L}}{\delta u}=v_{t}-v_{x x x}-v_{x} u-\bar{v}_{x} \bar{u}=0 \\
F_{2}^{*}=\frac{\delta \mathcal{L}}{\delta \bar{u}}=\bar{v}_{t}-\bar{v}_{x x x}-u \bar{v}_{x}-\varepsilon \bar{u} v_{x}=0 .
\end{array}\right.
$$

Obviously, (9) becomes equivalent to the Frobenius KDV Equation (6) upon the substituting

$$
v=\bar{u}, \bar{v}=u
$$

Thus, the Frobenius KDV equation is quasi self-adjoint. 
Example 2. The Frobenius KP equation (see [19])

$$
\left\{\begin{array}{l}
u_{t x}-u u_{x x}-\varepsilon \bar{u}_{x x}-u_{x}^{2}-\varepsilon \bar{u}_{x}^{2}-u_{x x x x}-u_{y y}=0 \\
\bar{u}_{t x}-\bar{u} u_{x x}-u \bar{u}_{x x}--u_{x} \bar{u}_{x}-\bar{u}_{x x x x}-\bar{u}_{y y}=0
\end{array}\right.
$$

which has an equivalent system as follows:

$$
\left\{\begin{array}{l}
F_{1}=u_{t}-u u_{x}-\varepsilon \overline{u u}_{x}-u_{x x x}-\omega_{y}=0, F_{2}=\omega_{x}-u_{y}=0 \\
F_{3}=\bar{u}_{t}-\bar{u} u_{x}-u \bar{u}_{x}-\bar{u}_{x x x}-\bar{\omega}_{y}=0, F_{4}=\bar{\omega}_{x}-\bar{u}_{y}=0
\end{array}\right.
$$

It follows that we obtain the adjoint system

$$
\left\{\begin{array}{l}
F_{1}^{*}=\frac{\delta \mathcal{L}}{\delta u}=-v_{1, t}+v_{1, x x x}+v_{1, x} u+\bar{v}_{1, x} \bar{u}+v_{2, y}=0, F_{2}^{*}=\frac{\delta \mathcal{L}}{\delta \omega}=v_{1, y}-v_{2, x} \\
F_{3}^{*}=\frac{\delta \mathcal{L}}{\delta \bar{u}}=-\bar{v}_{1, t}+\bar{v}_{1, x x x}+\bar{v}_{1, x} u+\varepsilon v_{1, x} \bar{u}+\bar{v}_{2, y}=0, F_{4}^{*}=\frac{\delta \mathcal{L}}{\delta \bar{\omega}}=\bar{v}_{1, y}-\bar{v}_{2, x}
\end{array}\right.
$$

with the formal Lagrangian

$$
\mathcal{L}=v_{1}\left(u_{t}-u u_{x}-\varepsilon \bar{u} \bar{u}_{x}-u_{x x x}-\omega_{y}\right)+v_{2}\left(\omega_{x}-u_{y}\right)+\bar{v}_{1}\left(\bar{u}_{t}-\bar{u} u_{x}-u \bar{u}_{x}-\bar{u}_{x x x}-\bar{\omega}_{y}\right)+\bar{v}_{2}\left(\bar{\omega}_{x}-\bar{u}_{y}\right) .
$$

In (13), we substitute

$$
v_{1}=\bar{u}, v_{2}=\bar{\omega}, \bar{v}_{1}=u, \bar{v}_{2}=\omega,
$$

then we obtain the Frobenius KP system (12). Therefore, the Frobenius KP equation is also quasi self-adjoint.

\section{Nonlinear Self-Adjointness of Frobenius Type Equations}

Definition 3. If the adjoint Equation (2) is satisfied for all solutions $u$ and $\bar{u}$ of the system (1) upon a substitution (see $[13,14])$

$$
v^{\alpha}=\varphi^{\alpha}(x, u), \bar{v}^{\alpha}=\bar{\varphi}^{\alpha}(x, \bar{u}), \alpha=1, \ldots, m
$$

such that

$$
\varphi^{\alpha}(x, u) \neq 0, \bar{\varphi}^{\alpha}(x, \bar{u}) \neq 0,
$$

then we call the Frobenius system (1) is nonlinearly self-adjoint.

It means that

$$
F_{\alpha}^{*}\left(x, u, \varphi(x, u), \bar{u}, \bar{\varphi}(x, \bar{u}), \ldots, u_{(s)}, \varphi_{(s)}, \bar{u}_{(s)}, \bar{\varphi}_{(s)}\right)=\lambda_{\alpha}^{\beta} F_{\beta}\left(x, u, \bar{u}_{,} \ldots, u_{(s)}, \bar{u}_{(s)}\right), \alpha=1, \ldots, m,
$$

where

$\varphi=\left\{\varphi^{\alpha}(x, u)\right\}, \bar{\varphi}=\left\{\bar{\varphi}^{\alpha}(x, \bar{u})\right\}, \varphi_{k}=\left\{D_{i} \ldots D_{k}\left(\varphi^{\alpha}(x, u)\right)\right\}, \bar{\varphi}_{k}=\left\{D_{i} \ldots D_{k}\left(\bar{\varphi}^{\alpha}(x, \bar{u})\right)\right\}, k=1, \ldots, s$.

Proposition 1. The nonlinearly self-adjointness of Equation (1) is equivalent to the following statement: Equation (1) can become strictly self-adjoint if we rewrite it as: (see $[13,14])$

$$
\begin{aligned}
& \mu^{\alpha}(x, u) F_{\alpha}\left(x, u, \bar{u}, u_{(1)}, \bar{u}_{(1)}, \ldots, u_{(s)}, \bar{u}_{(s)}\right)=0, \\
& \bar{\mu}^{\beta}(x, \bar{u}) F_{\beta}\left(x, u, \bar{u}, u_{(1)}, \bar{u}_{(1)}, \ldots, u_{(s)}, \bar{u}_{(s)}\right)=0, \\
& \mu^{\alpha}(x, u) \neq 0, \bar{\mu}^{\beta}(x, \bar{u}) \neq 0, \alpha=1, \ldots, m, \beta=1, \ldots, m,
\end{aligned}
$$

with the appropriate multipliers $\mu^{\alpha}(x, u)$ and $\bar{\mu}^{\beta}(x, \bar{u})$. 
In the following, we will give an example of the Frobenius Equation (1) that is not quasi self-adjoint, but nonlinearly self-adjoint. The classical Kompaneets equation has the following form (see [26])

$$
\begin{aligned}
u_{t} & =\frac{1}{x^{2}} D_{x}\left[x^{4}\left(u_{x}+u+u^{2}\right)\right] \\
& =x^{2} u_{x x}+\left(x^{2}+4 x+2 x^{2} u\right) u_{x}+4 x\left(u+u^{2}\right) .
\end{aligned}
$$

Based on the knowledge of Frobenius algebra (see [19-23,25]), we introduce the Frobenius Kompaneets equation as shown in the following example.

Example 3. The Frobenius Kompaneets equation

$$
\left\{\begin{array}{l}
u_{t}=x^{2} u_{x x}+\left(x^{2}+4 x+2 x^{2} u\right) u_{x}+2 \varepsilon x^{2} \overline{u u}_{x}+4 x\left(u+u^{2}+\varepsilon \bar{u}^{2}\right) \\
\bar{u}_{t}=x^{2} \bar{u}_{x x}+\left(x^{2}+4 x+2 x^{2} u\right) \bar{u}_{x}+2 x^{2} \bar{u} u_{x}+4 x(\bar{u}+2 u \bar{u})
\end{array}\right.
$$

is nonlinearly self-adjoint.

The formal Lagrangian of the Frobenius Kompaneets Equation (18) is given by

$$
\begin{aligned}
\mathcal{L}= & v\left(-u_{t}+x^{2} u_{x x}+\left(x^{2}+4 x+2 x^{2} u\right) u_{x}+2 \varepsilon x^{2} \overline{u u}_{x}+4 x\left(u+u^{2}+\varepsilon \bar{u}^{2}\right)\right)+ \\
& \bar{v}\left(-\bar{u}_{t}+x^{2} \bar{u}_{x x}+\left(x^{2}+4 x+2 x^{2} u\right) \bar{u}_{x}+2 x^{2} \bar{u} u_{x}+4 x(\bar{u}+2 u \bar{u})\right)
\end{aligned}
$$

presents

$$
\left\{\begin{array}{l}
\frac{\delta \mathcal{L}}{\delta u}=v_{t}+x^{2} v_{x x}-x^{2}(1+2 u) v_{x}+2(x+2 x u-1) v+4 x \overline{u v}-2 x^{2} \overline{u v}_{x}=0 \\
\frac{\delta \mathcal{L}}{\delta \bar{u}}=\bar{v}_{t}+x^{2} \bar{v}_{x x}-x^{2}(1+2 u) \bar{v}_{x}+2(x+2 x u-1) \bar{v}+4 \varepsilon x \bar{u} v-2 \varepsilon x^{2} \bar{u} v_{x}=0 .
\end{array}\right.
$$

It is not difficult to verify that the self-adjointness condition (5)

$$
\left\{\begin{aligned}
\frac{\delta \mathcal{L}}{\delta u}= & \lambda_{1}\left(-u_{t}+x^{2} u_{x x}+\left(x^{2}+4 x+2 x^{2} u\right) u_{x}+2 \varepsilon x^{2} \overline{u u}_{x}+4 x\left(u+u^{2}+\varepsilon \bar{u}^{2}\right)\right) \\
& +\lambda_{2}\left(-\bar{u}_{t}+x^{2} \bar{u}_{x x}+\left(x^{2}+4 x+2 x^{2} u\right) \bar{u}_{x}+2 x^{2} \bar{u} u_{x}+4 x(\bar{u}+2 u \bar{u})\right), \\
\frac{\delta \mathcal{L}}{\delta \bar{u}}= & \lambda_{3}\left(-u_{t}+x^{2} u_{x x}+\left(x^{2}+4 x+2 x^{2} u\right) u_{x}+2 \varepsilon x^{2} \overline{u u}_{x}+4 x\left(u+u^{2}+\varepsilon \bar{u}^{2}\right)\right) \\
& +\lambda_{4}\left(-\bar{u}_{t}+x^{2} \bar{u}_{x x}+\left(x^{2}+4 x+2 x^{2} u\right) \bar{u}_{x}+2 x^{2} \bar{u} u_{x}+4 x(\bar{u}+2 u \bar{u})\right),
\end{aligned}\right.
$$

is not satisfied upon the substitution $v=\varphi(u)$ and $\bar{v}=\bar{\varphi}(\bar{u})$. Hence, the Frobenius Kompaneets equation is not quasi self-adjoint. However, the Frobenius Kompaneets equation satisfy the nonlinearly self-adjoint condition (17) via the substitution $v=\varphi(t, x, u)$ and $\bar{v}=\bar{\varphi}(t, x, \bar{u})$. In the following, we give the detailed argument. In this case, the derivatives of $v$ and $\bar{v}$ reads

$$
\begin{aligned}
& v=\varphi(t, x, u), \bar{v}=\bar{\varphi}(t, x, \bar{u}), \\
& v_{t}=D_{t}[\varphi(t, x, u)]=\varphi_{u} u_{t}+\varphi_{t}, v_{x}=D_{x}[\varphi(t, x, u)]=\varphi_{u} u_{x}+\varphi_{x}, \\
& v_{x x}=D_{x}\left(v_{x}\right)=\varphi_{u} u_{x x}+\varphi_{u u} u_{x}^{2}+2 \varphi_{x u} u_{x}+\varphi_{x x}, \\
& \bar{v}_{t}=D_{t}[\bar{\varphi}(t, x, \bar{u})]=\bar{\varphi}_{\bar{u}} \bar{u}_{t}+\bar{\varphi}_{t}, \bar{v}_{x}=D_{x}[\bar{\varphi}(t, x, \bar{u})]=\bar{\varphi}_{\bar{u}} \bar{u}_{x}+\bar{\varphi}_{x}, \\
& \bar{v}_{x x}=D_{x}\left(\bar{v}_{x}\right)=\bar{\varphi}_{\bar{u}} \bar{u}_{x x}+\bar{\varphi}_{\overline{u u}} \bar{u}_{x}^{2}+2 \bar{\varphi}_{x \bar{u}} \bar{u}_{x}+\bar{\varphi}_{x x} .
\end{aligned}
$$

Substituting (22) into Equation (20) and singling out in Equation (21) the terms containing $u_{t}$, $\bar{u}_{t}, u_{x x}$ and $\bar{u}_{x x}$, we have

$$
\lambda_{1}=-\varphi_{u}, x^{2} \lambda_{1}=x^{2} \varphi_{u}, \lambda_{2}=-\bar{\varphi}_{\bar{u}}, x^{2} \lambda_{2}=x^{2} \bar{\varphi}_{\bar{u}}, \lambda_{3}=\lambda_{4}=0
$$


Thus,

$$
\lambda_{1}=\lambda_{2}=\lambda_{3}=\lambda_{4}=\varphi_{u}=\bar{\varphi}_{\bar{u}}=0,
$$

leads to $\varphi=\varphi(t, x), \bar{\varphi}=\bar{\varphi}(t, x)$, and then (21) becomes

$$
\left\{\begin{array}{l}
\varphi_{t}+x^{2} \varphi_{x x}-x^{2}(1+2 u) \varphi_{x}+2(x+2 x u-1) \varphi+4 x \bar{u} \bar{\varphi}-2 x^{2} \bar{u}_{x}=0, \\
\bar{\varphi}_{t}+x^{2} \bar{\varphi}_{x x}-x^{2}(1+2 u) \bar{\varphi}_{x}+2(x+2 x u-1) \bar{\varphi}+4 \varepsilon x \bar{u} \varphi-2 \varepsilon x^{2} \bar{u} \varphi_{x}=0 .
\end{array}\right.
$$

Comparing the coefficients of $u$ and $\bar{u}$ yields

$$
x \varphi_{x}-2 \varphi=0, x \bar{\varphi}_{x}-2 \bar{\varphi}=0,
$$

we thus obtain

$$
\varphi(t, x)=\bar{\varphi}(t, x)=c(t) x^{2} .
$$

Then, one can find that $\frac{d c}{d t}=0$ by substituting (24) into (23). We further obtain

$$
v=\varphi(t, x)=C x^{2}, \bar{v}=\bar{\varphi}(t, x)=C x^{2},
$$

with constant $C$. Therefore, we have demonstrated the Frobenius Kompaneets equation is nonlinearly self-adjoint.

Remark 1. The Equation (25) shows that $v$ does not depend on $u$ and $\bar{v}$ does not depend on $\bar{u}$. However, The question arises on existence of a substitution $v=\varphi(t, x, u)$ and $\bar{v}=\bar{\varphi}(t, x, \bar{u})$ involving $u$ and $\bar{u}$ if we rewrite (18) in an equivalent form (see proposition 1)

$$
\left\{\begin{aligned}
\frac{x^{2}}{u} u_{t} & =\frac{x^{2}}{u}\left[x^{2} u_{x x}+\left(x^{2}+4 x+2 x^{2} u\right) u_{x}+2 \varepsilon x^{2} \bar{u}_{x}+4 x\left(u+u^{2}+\varepsilon \bar{u}^{2}\right)\right] \\
& =\frac{1}{u} D_{x}\left[x^{4}\left(u_{x}+u+u^{2}+\varepsilon \bar{u}^{2}\right)\right] \\
\frac{x^{2}}{\bar{u}} \bar{u}_{t} & =\frac{x^{2}}{\bar{u}}\left[x^{2} \bar{u}_{x x}+\left(x^{2}+4 x+2 x^{2} u\right) \bar{u}_{x}+2 x^{2} \bar{u} u_{x}+4 x(\bar{u}+2 u \bar{u})\right] \\
& =\frac{1}{\bar{u}} D_{x}\left[x^{4}\left(u_{x}+u+2 u \bar{u}\right)\right]
\end{aligned}\right.
$$

with the multipliers $\mu(t, x, u)=\frac{x^{2}}{u}$ and $\bar{\mu}(t, x, \bar{u})=\frac{x^{2}}{\bar{u}}$.

Example 4. For the classical Burgers equation

$$
u_{t}-u_{x x}-u u_{x}=0,
$$

we introduce the Frobenius Burgers equation as follows:

$$
\left\{\begin{array}{l}
F_{1}=u_{t}-u_{x x}-u u_{x}-\varepsilon \overline{u u}_{x}=0, \\
F_{2}=\bar{u}_{t}-\bar{u}_{x x}-\bar{u} u_{x}-u \bar{u}_{x}=0,
\end{array}\right.
$$

which is nonlinearly self-adjoint via Multipliers.

Taking the multipliers $\mu=\frac{1}{u}$ and $\bar{\mu}=\frac{1}{\bar{u}}$ so that for

$$
\left\{\begin{array}{l}
\frac{1}{u}\left(u_{t}-u_{x x}-u u_{x}-\varepsilon \overline{u u}_{x}\right)=0, \\
\frac{1}{\bar{u}}\left(\bar{u}_{t}-\bar{u}_{x x}-\bar{u} u_{x}-u \bar{u}_{x}\right)=0 .
\end{array}\right.
$$


Then, the adjoint system of Equation (28) is obtained as follows:

$$
\left\{\begin{array}{l}
\frac{\delta \mathcal{L}}{\delta u}=-\frac{v}{u^{2}}\left(u_{t}-u_{x x}-u u_{x}-\varepsilon \overline{u u}_{x}\right)-\frac{v u_{x}}{u}-\frac{\bar{v} \bar{u}_{x}}{\bar{u}}+D_{x}(v)+D_{x}(\bar{v})-D_{t}\left(\frac{v}{u}\right)-D_{x}^{2}\left(\frac{v}{u}\right), \\
\frac{\delta \mathcal{L}}{\delta \bar{u}}=-\frac{\bar{v}}{\bar{u}^{2}}\left(\bar{u}_{t}-\bar{u}_{x x}-\bar{u} u_{x}-u \bar{u}_{x}\right)-\varepsilon \frac{v \bar{u} x_{x}}{u}-\frac{\bar{v} u_{x}}{\bar{u}}+\varepsilon \frac{v}{u} D_{x}(\bar{u})+\frac{\bar{v}}{\bar{u}} D_{x}(u)-D_{t}\left(\frac{\bar{v}}{\bar{u}}\right)-D_{x}^{2}\left(\frac{\bar{v}}{\bar{u}}\right),
\end{array}\right.
$$

with

$$
\mathcal{L}=\frac{v}{u}\left(u_{t}-u_{x x}-u u_{x}-\varepsilon \overline{u u}_{x}\right)+\frac{\bar{v}}{\bar{u}}\left(\bar{u}_{t}-\bar{u}_{x x}-\bar{u} u_{x}-u \bar{u}_{x}\right) .
$$

Substituting $v=u$ and $\bar{v}=\bar{u}$ into (29), one has

$$
\left\{\begin{array}{l}
\frac{\delta \mathcal{L}}{\delta u}=-\frac{1}{u}\left(u_{t}-u_{x x}-u u_{x}-\varepsilon \overline{u u}_{x}\right) \\
\frac{\delta \mathcal{L}}{\delta \bar{u}}=-\frac{1}{\bar{u}}\left(\bar{u}_{t}-\bar{u}_{x x}-\bar{u} u_{x}-u \bar{u}_{x}\right)
\end{array}\right.
$$

By comparing (31) and (28), we find that Equation (28) is strictly self-adjoint. Thus, the Frobenius Burgers equation is nonlinearly self-adjoint.

Example 5. To the Harry Dym equation

$$
u_{t}-u^{3} u_{x x x}=0
$$

we introduce its corresponding Frobenius type equation as follows:

$$
\left\{\begin{array}{l}
F_{1}=u_{t}-u_{x x x}\left(u^{3}+3 \varepsilon u \bar{u}^{2}\right)-\varepsilon \bar{u}_{x x x}\left(\varepsilon \bar{u}^{3}+3 u^{2} \bar{u}\right)=0, \\
F_{2}=\bar{u}_{t}-u_{x x x}\left(\varepsilon \bar{u}^{3}+3 u^{2} \bar{u}\right)-\bar{u}_{x x x}\left(u^{3}+3 \varepsilon u \bar{u}^{2}\right)=0,
\end{array}\right.
$$

which is also nonlinearly self-adjoint.

Similarly, we take the multipliers $\mu=\frac{1}{u^{4}}$ and $\bar{\mu}=\frac{1}{\bar{u}^{4}}$ so that for

$$
\left\{\begin{array}{l}
\frac{1}{u^{4}}\left[u_{t}-u_{x x x}\left(u^{3}+3 \varepsilon u \bar{u}^{2}\right)-\varepsilon \bar{u}_{x x x}\left(\varepsilon \bar{u}^{3}+3 u^{2} \bar{u}\right)\right]=0 \\
\frac{1}{\bar{u}^{4}}\left[\bar{u}_{t}-u_{x x x}\left(\varepsilon \bar{u}^{3}+3 u^{2} \bar{u}\right)-\bar{u}_{x x x}\left(u^{3}+3 \varepsilon u \bar{u}^{2}\right)\right]=0
\end{array}\right.
$$

The formal Lagrangian for Equation (33) has the form as follows:

$$
\mathcal{L}=\frac{v}{u^{4}}\left[u_{t}-u_{x x x}\left(u^{3}+3 \varepsilon u \bar{u}^{2}\right)-\varepsilon \bar{u}_{x x x}\left(\varepsilon \bar{u}^{3}+3 u^{2} \bar{u}\right)\right]+\frac{\bar{v}}{\bar{u}^{4}}\left[\bar{u}_{t}-u_{x x x}\left(\varepsilon \bar{u}^{3}+3 u^{2} \bar{u}\right)-\bar{u}_{x x x}\left(u^{3}+3 \varepsilon u \bar{u}^{2}\right)\right],
$$

which admits that

$$
\left\{\begin{array}{l}
\left.\frac{\delta \mathcal{L}}{\delta u}\right|_{v=u, \bar{v}=\bar{u}}=-F_{1} \\
\left.\frac{\delta \mathcal{L}}{\delta \bar{u}}\right|_{v=u, \bar{v}=\bar{u}}=-F_{2}
\end{array}\right.
$$

Thus, the Frobenius Harry Dym equation is nonlinearly self-adjoint.

\section{Conservation Laws of Frobenius Type Equations}

In Sections 2 and 3, we discussed the quasi self-adjointness and nonlinear self-adjointness of the Frobenius type equation respectively. After exploring the self-adjointness, we can construct the conservation law of the Frobenius type equation by means of symmetry. In this section, we will consider the symmetry and conservation laws of the Frobenius KDV Equation (6) and the (2+1)-dimensional Frobenius KP system (12) as two examples based on the following theorem. 
Theorem 1. To the Frobenius Equation (1), the conservation law $D_{i}\left(C^{i}\right)=0$ can be constructed by any Lie point, Lie-Bäcklund, and nonlocal symmetry (see [12-16])

$$
X=\xi^{i}\left(x, u, u_{(1)}, \ldots\right) \frac{\partial}{\partial x^{i}}+\eta^{a}\left(x, u, u_{(1)}, \ldots\right) \frac{\partial}{\partial u^{a}},
$$

where

$$
\begin{aligned}
C^{i}= & \xi^{i} \mathcal{L}+W^{\alpha}\left[\frac{\partial \mathcal{L}}{\partial u_{i}^{\alpha}}-D_{j}\left(\frac{\partial \mathcal{L}}{\partial u_{i j}^{\alpha}}\right)+D_{j} D_{k}\left(\frac{\partial \mathcal{L}}{\partial u_{i j k}^{\alpha}}\right)-\cdots\right]+D_{j}\left(W^{\alpha}\right)\left[\frac{\partial \mathcal{L}}{\partial u_{i j}^{\alpha}}-D_{k}\left(\frac{\partial \mathcal{L}}{\partial u_{i j k}^{\alpha}}\right)+\cdots\right] \\
& +D_{j} D_{k}\left(W^{\alpha}\right)\left[\frac{\partial \mathcal{L}}{\partial u_{i j k}^{\alpha}}-\cdots\right]+\cdots,
\end{aligned}
$$

with $W^{\alpha}=\eta^{\alpha}-\xi^{j} u_{j}^{\alpha}, \alpha=1, \ldots, m$.

With the aid of symbolic computation by Maple, the infinitesimal symmetry of the Frobenius KDV Equation (6) is obtained as follows:

$$
X=(2 \varepsilon \bar{u}-2 u) \frac{\partial}{\partial u}+(3 \varepsilon \bar{u}+u-4 \bar{u}) \frac{\partial}{\partial \bar{u}}+3 t \frac{\partial}{\partial t}+(x-3 \varepsilon t \bar{u}) \frac{\partial}{\partial x} .
$$

Eliminating $v$ and $\bar{v}$ in the formal Lagrangian (8) by the substituting (10), the symmetry $X$ yields the conservation law

$$
\left[D_{t}\left(C^{1}\right)+D_{x}\left(C^{2}\right)\right]_{(6)}=0,
$$

where the conserved vector $C=\left(C^{1}, C^{2}\right)$ is given by (36) and has the following components

$$
\begin{aligned}
C^{1}=\xi^{1} \mathcal{L}+W^{1}\left(\frac{\partial \mathcal{L}}{\partial u_{t}}\right)+W^{2}\left(\frac{\partial \mathcal{L}}{\partial \bar{u}_{t}}\right) & \\
= & x u \bar{u}_{x}+x \bar{u} u_{x}+3 t \bar{u} u_{x x x}+3 t u \bar{u}_{x x x}+6 t u \bar{u} u_{x}+3 \varepsilon t \bar{u}^{2} \bar{u}_{x}-3 \varepsilon t \bar{u}^{2} u_{x} \\
& +3 t u^{2} \bar{u}_{x}-3 \varepsilon t u \bar{u} \bar{u}_{x}-2 \varepsilon \bar{u}^{2}-3 \varepsilon u \bar{u}+6 u \bar{u}-u^{2}, \\
C^{2}= & \xi^{2} \mathcal{L}+W^{1}\left[\frac{\partial \mathcal{L}}{\partial u_{x}}+D_{x}^{2}\left(\frac{\partial \mathcal{L}}{\partial u_{x x x}}\right)\right]+D_{x}^{2}\left(W^{1}\right)\left(\frac{\partial \mathcal{L}}{\partial u_{x x x}}\right)+W^{2}\left[\frac{\partial \mathcal{L}}{\partial \bar{u}_{x}}+D_{x}^{2}\left(\frac{\partial \mathcal{L}}{\partial \bar{u}_{x x x}}\right)\right]+D_{x}^{2}\left(W^{2}\right)\left(\frac{\partial \mathcal{L}}{\partial \bar{u}_{x x x}}\right) \\
= & u\left(-12 \varepsilon t \bar{u}^{2} u_{x}-x \bar{u}_{t}+3 \varepsilon t \bar{u} \bar{u}_{t}+5 \varepsilon \bar{u}^{2}-8 u \bar{u}-8 \bar{u}_{x x}+2 u_{x x}-6 t \bar{u} u_{t}-3 t u \bar{u} t+3 \varepsilon u \bar{u}\right. \\
+ & \left.u^{2}-3 t \bar{u}_{x x t}+9 \varepsilon t \bar{u}_{x} \bar{u}_{x x}+3 \varepsilon \bar{u}_{x x}\right)+\bar{u}\left(-x u_{t}+3 \varepsilon t \bar{u} u_{t}-3 \varepsilon t \bar{u} \bar{u}_{t}+9 \varepsilon t \bar{u}_{x} u_{x x}\right. \\
+ & \left.4 \varepsilon \bar{u}_{x x}+6 \varepsilon t u_{x} \bar{u}_{x x}+3 \varepsilon^{2} \bar{u}^{2}-4 \varepsilon \bar{u}^{2}+3 \varepsilon u_{x x}-8 u_{x x}-3 t u_{x x t}\right) \\
+ & u_{x x}\left(-x \bar{u}_{x}-3 t \bar{u}_{t}\right)+\bar{u}_{x x}\left(-x u_{x}-3 t u_{t}\right)
\end{aligned}
$$

with

$$
W^{1}=2 \varepsilon \bar{u}-2 u-3 t u_{t}-(x-3 \varepsilon t \bar{u}) u_{x}, \quad W^{2}=3 \varepsilon \bar{u}+u-4 \bar{u}-3 t \bar{u}_{t}-(x-3 \varepsilon t \bar{u}) \bar{u}_{x} .
$$

Similarly, the Frobenius KP system (12) admits the following three symmetries

$$
\begin{aligned}
& X_{1}=-f^{\prime \prime} y \frac{\partial}{\partial u}-\left(u f^{\prime}+f^{\prime \prime} x+\frac{1}{2} f^{\prime \prime \prime} y^{2}\right) \frac{\partial}{\partial \omega}-f^{\prime} \bar{u} \frac{\partial}{\partial \bar{\omega}}+f^{\prime} y \frac{\partial}{\partial x}+2 f \frac{\partial}{\partial y^{\prime}} \\
& X_{2}=-g^{\prime} \frac{\partial}{\partial u}+g \frac{\partial}{\partial x}-g^{\prime \prime} y \frac{\partial}{\partial \omega}, \\
& X_{3}=-2 u \frac{\partial}{\partial u}-4 \bar{u} \frac{\partial}{\partial \bar{u}}-3 \omega \frac{\partial}{\partial \omega}-5 \bar{\omega} \frac{\partial}{\partial \bar{\omega}}+x \frac{\partial}{\partial x}+2 y \frac{\partial}{\partial y}+3 t \frac{\partial}{\partial t^{\prime}}
\end{aligned}
$$

where $f$ and $g$ are arbitrary functions of $t$, i.e., $f=f(t), g=g(t)$. In the following, we mainly discuss the second symmetry $X_{2}$ and the third symmetry $X_{3}$ of the Frobenius KP system in (38). 
Eliminating $v_{1}, v_{2}, \bar{v}_{1}$ and $\bar{v}_{2}$ in the formal Lagrangian (14) by the substituting (15), the symmetry $\mathrm{X}_{2}$ admits

$$
\left[D_{t}\left(C^{1}\right)+D_{x}\left(C^{2}\right)+D_{y}\left(C^{3}\right)\right]_{(12)}=0
$$

with the following components of the conserved vector $C=\left(C^{1}, C^{2}, C^{3}\right)$ :

$$
\begin{gathered}
C^{1}=W^{1}\left(\frac{\partial \mathcal{L}}{\partial u_{t}}\right)=-g^{\prime} \bar{u}-g u_{x} \bar{u}, \\
C^{2}=\bar{\zeta}^{2} \mathcal{L}+W^{1}\left[\frac{\partial \mathcal{L}}{\partial u_{x}}+D_{x}^{2}\left(\frac{\partial \mathcal{L}}{\partial u_{x x x}}\right)\right]+D_{x}^{2}\left(W^{1}\right)\left(\frac{\partial \mathcal{L}}{\partial u_{x x x}}\right)+W^{2}\left(\frac{\partial \mathcal{L}}{\partial \omega_{x}}\right) \\
=g u\left(\bar{u}_{t}-u \bar{u}_{x}-\bar{u}_{x x x}-\bar{\omega}_{y}\right)+g \bar{u}\left(u_{t}-\varepsilon \bar{u} \bar{u}_{x}-\omega_{y}\right) \\
+g \omega\left(\bar{\omega}_{x}-\bar{u}_{y}\right)+\bar{\omega}\left(-g u_{y}-g^{\prime \prime} y\right)+\bar{u}_{x x}\left(g^{\prime}+g u_{x}\right)+2 g^{\prime} u \bar{u}, \\
C^{3}=W^{1}\left(\frac{\partial \mathcal{L}}{\partial u_{y}}\right)+W^{2}\left(\frac{\partial \mathcal{L}}{\partial \omega_{y}}\right)=g^{\prime \prime} y \bar{u}+g^{\prime} \bar{\omega}+g\left(u_{x} \bar{\omega}+\omega_{x} \bar{u}\right),
\end{gathered}
$$

where

$$
W^{1}=-g^{\prime}-g u_{x}, W^{2}=-g^{\prime \prime} y-g \omega_{x}
$$

To the symmetry $X_{3}$, we eliminate $v_{1}, v_{2}, \bar{v}_{1}$ and $\bar{v}_{2}$ in Equation (14) by substituting (15), one has

$$
\left[D_{t}\left(C^{1}\right)+D_{x}\left(C^{2}\right)+D_{y}\left(C^{3}\right)\right]_{(12)}=0,
$$

where

$$
\begin{aligned}
& C^{1}=\tilde{\zeta}^{1} \mathcal{L}+W^{1}\left(\frac{\partial \mathcal{L}}{\partial u_{t}}\right)+W^{2}\left(\frac{\partial \mathcal{L}}{\partial \bar{u}_{t}}\right) \\
& =u\left(-6 t u_{x} \bar{u}-3 t \bar{u}_{x x x}-3 t \bar{\omega}_{y}-3 t u \bar{u}_{x}-6 \bar{u}-2 y \bar{u}_{y}-x \bar{u}_{x}\right)+\bar{u}\left(-3 \varepsilon t \bar{u} \bar{u}_{x}\right. \\
& \left.-3 t u_{x x x}-3 t \omega_{y}-2 y u_{y}-x \omega_{x}\right)+\omega\left(3 t \bar{u}_{y}-3 t \bar{\omega}_{x}\right)+\bar{\omega}\left(3 t \omega_{x}-3 t u_{y}\right), \\
& C^{2}=\tilde{\xi}^{2} \mathcal{L}+W^{1}\left[\frac{\partial \mathcal{L}}{\partial u_{x}}+D_{x}^{2}\left(\frac{\partial \mathcal{L}}{\partial u_{x x x}}\right)\right]+D_{x}^{2}\left(W^{1}\right)\left(\frac{\partial \mathcal{L}}{\partial u_{x x x}}\right)+W^{2}\left[\frac{\partial \mathcal{L}}{\partial \bar{u}_{x}}+D_{x}^{2}\left(\frac{\partial \mathcal{L}}{\partial \bar{u}_{x x x}}\right)\right] \\
& +D_{x}^{2}\left(W^{2}\right)\left(\frac{\partial \mathcal{L}}{\partial \bar{u}_{x x x}}\right)+W^{3}\left(\frac{\partial \mathcal{L}}{\partial \omega_{x}}\right)+W^{4}\left(\frac{\partial \mathcal{L}}{\partial \bar{\omega}_{x}}\right) \\
& =u\left(-x \bar{\omega}_{y}+8 \bar{u}+8 \bar{u}_{x x}+3 t \bar{u}_{x x t}+3 y \bar{u}_{x x y}+6 u \bar{u} u_{t}+4 y \bar{u} u_{y}+x \bar{u}_{t}+3 t u \bar{u}_{t}+2 y \bar{u}_{y}\right) \\
& +\bar{u}\left(x u_{t}+3 \varepsilon t \overline{u u}_{t}+2 \varepsilon y \overline{u u}_{y}\right)+\omega\left(-8 \bar{\omega}-3 t \bar{\omega}_{t}-2 y \omega_{y}-x \omega_{x}+x \bar{\omega}_{x}-x \bar{u}_{y}\right) \\
& +\bar{\omega}\left(-3 t \omega_{t}-2 y \omega_{y}-x u_{y}\right)+u_{x x}\left(x \bar{u}_{x}+3 t+2 y \bar{u}_{y}\right)+\bar{u}_{x x}\left(x u_{x}+3 t+2 y u_{y}\right), \\
& C^{3}=\xi^{3} \mathcal{L}+W^{1}\left(\frac{\partial \mathcal{L}}{\partial u_{y}}\right)+W^{2}\left(\frac{\partial \mathcal{L}}{\partial \bar{u}_{y}}\right)+W^{3}\left(\frac{\partial \mathcal{L}}{\partial \omega_{y}}\right)+W^{4}\left(\frac{\partial \mathcal{L}}{\partial \bar{\omega}_{y}}\right) \\
& =u\left(7 \bar{\omega}+3 t \bar{\omega}_{t}+x \omega_{x}-2 y u u_{x}-4 y \bar{u} u_{x}+2 y \bar{u}_{t}-2 y \bar{u}_{x x x}\right)+\bar{u}\left(7 \omega_{t}+3 t \omega_{t}+x \omega_{x}+2 y u_{t}+2 \varepsilon y \bar{u} \bar{u}_{x}\right) \\
& +\omega\left(x \bar{u}_{x}+3 t \bar{u}_{t}+2 y \bar{\omega}_{x}\right)+\bar{\omega}\left(x u_{x}+3 t u_{t}+2 y \omega_{x}\right),
\end{aligned}
$$

with

$$
\begin{aligned}
& W^{1}=-2 u-3 t u_{t}-2 y u_{y}-x u_{x}, \quad W^{2}=-4 \bar{u}-3 t \bar{u}_{t}-2 y \bar{u}_{y}-x \bar{u}_{x} \\
& W^{3}=-3 \omega-3 t \omega_{t}-2 y \omega_{y}-x \omega_{x}, \quad W^{4}=-5 \bar{\omega}-3 t \bar{\omega}_{t}-2 y \omega_{y}-x \omega_{x} .
\end{aligned}
$$

\section{Conclusions and Discussion}

In this paper, the Frobenius KDV equation and Frobenius KP equation are introduced as two examples in order to study the quasi self-adjointness of the Frobenius type equation. 
Then, the Frobenius Kompaneets equation, Frobenius Burgers equation and Frobenius Harry Dym equation are constructed which is defined by us through taking values in a commutative subalgebra $\mathcal{Z}_{2}^{\varepsilon}$, and the three equation can be used to investigate the nonlinear self-adjointness of the Frobenius type equation. Investigating the conservation laws is critical to study the integrability of optimal system, we thus construct the conservation laws of the Frobenius KDV equation and the $(2+1)$-dimensional Frobenius KP system as two examples via the direct method. The idea and the method used here are universal for other Frobenius equations. The paper focus on the Frobenius equation of type $\mathcal{Z}_{2}^{\varepsilon}$. Actually, the self-adjointness and the conservation laws of $\mathcal{Z}_{n}$ Frobenius equation may also be discussed in our future work (see [21,22,24,27]). Additionally, the time-fractional Frobenius equation $($ see $[17,28])$ and some exact solutions (see $[16,17])$ can also be considered in the forthcoming days.

Author Contributions: Conceptualization, H.W.; formal analysis, Y.Z.; methodology, H.W.; software, Y.Z.; writing, original draft, H.W.; writing, review and editing, H.W. All authors have read and agreed to the published version of the manuscript.

Funding: This work is supported by the Future Scientists Program of China University of Mining and Technology (No.2020WLKXJ036) and the Postgraduate Research \& Practice Innovation Program of Jiangsu Province (No.KYCX20_1975).

Conflicts of Interest: The authors declare no conflict of interest.

\section{References}

1. Gardner, C.S.; Greene, J.M.; Kruskal, M.D.; Miura, R.M. Method for solving the Korteweg-de Vries equation. Phys. Rev. Lett. 1967, 19, 1095-1097. [CrossRef]

2. Olver, P.J. Applications of Lie Groups to Differential Equations; Springer Science and Business Media: New York, NY, USA, 2012.

3. Wang, Y.H. CTE method to the interaction solutions of Boussinesq-Burgers equations. Appl. Math. Lett. 2014, 38, 100-105. [CrossRef]

4. Esipov, S.E. Coupled Burgers equations: A model of polydispersive sedimentation. Phys. Rev. E 1995, 52, 3711-3718. [CrossRef] [PubMed]

5. Zhang, Y.F.; Fan, E.G.; Tam, H.W. A few expanding Lie algebras of the Lie algebra $A_{1}$ and applications. Phys. Lett. A 2006, 359, 471-480. [CrossRef]

6. Zhang, Y.F.; Tam, H.W. Discussion on integrable properties for higher-dimensional variable-coefficient nonlinear partial differential equations. J. Math. Phys. 2013, 54, 013516. [CrossRef]

7. Naz, R.; Mason, D.P.; Mahomed, F.M. Conservation laws and conserved quantities for laminar two-dimensional and radial jets. Nonlinear Anal. Real 2009, 10, 2641-2651. [CrossRef]

8. Bokhari, A.H.; Al-Dweik, A.Y.; Kara, A.H.; Mahomed, F.M.; Zaman, F.D. Double reduction of anonlinear $(2+1)$ wave equation via conservation laws. Commun. Nonlinear Sci. 2011, 16, 1244-1253. [CrossRef]

9. Avdonina, E.D.; Ibragimov, N.H.; Khamitova, R. Exact solutions of gasdynamic equations obtained by the method of conservation laws. Commun. Nonlinear Sci. 2013, 18, 2359-2366. [CrossRef]

10. Noether, E. Invariante Variationsprobleme; Königliche Gesellschaft der Wissenschaften zu Göttingen, Nachrichten: Gottingen, Germany, 1971; Volume 1, pp. 186-207.

11. Ibragimov, N.H. Integrating factors, adjoint equations and Lagrangians. J. Math. Anal. Appl. 2006, 318, 742-757. [CrossRef]

12. Ibragimov, N.H. A new conservation theorem. J. Math. Anal. Appl. 2007, 333, 311-328. [CrossRef]

13. Ibragimov, N.H. Nonlinear self-adjointness in constructing conservation laws. Arch. ALGA 2011, 7, 1-99.

14. Ibragimov, N.H. Nonlinear self-adjointness and conservation laws. J. Phys. A Math. Theor. 2011, 44, 432002. [CrossRef]

15. Ibragimov, N.H. Self-adjointness and conservation laws of a generalized Burgers equation. J. Phys. A Math. Theor. 2011, 44, 145201. [CrossRef]

16. Ibragimov, N.H. Conservation laws and non-invariant solutions of anisotropic wave equations with a source. Nonlinear Anal. Real 2018, 40, 82-94. [CrossRef]

17. Gazizov, R.K.; Ibragimov, N.H.; Lukashchuk, S.Y. Nonlinear self-adjointness, conservation laws and exact solutions of time-fractional Kompaneets equations. Commun. Nonlinear Sci. 2015, 23, 153-163. [CrossRef] 
18. Zhao, Z.L.; Han, B. On symmetry analysis and conservation laws of the AKNS system. Z. Naturforsch. 2016, 71, 741-750. [CrossRef]

19. Strachan, I.A.B.; Zuo, D.F. Integrability of the Frobenius algebra-valued Kadomtsev-Petviashvili hierarchy. J. Math. Phys. 2015, 56, 113509. [CrossRef]

20. Zuo, D.F. The Frobenius-Virasoro algebra and Euler equations. J. Geom. Phys. 2014, 86, 203-210. [CrossRef]

21. Li, C.Z.; He, J.S. The extended $\mathcal{Z}_{n}$-toda hierarchy. Theor. Math. Phys. 2015, 185, 1614-1635. [CrossRef]

22. Li, C.Z. Gauge transformation and symmetries of the commutative multi-component BKP hierarchy. J. Phys. A Math. Theor. 2016, 49, 015203. [CrossRef]

23. Wang, H.F.; Li, C.Z. Affine Weyl group symmetries of Frobenius Painlevé equations. Math. Meth. Appl. Sci. 2020, 43, 3238-3252. [CrossRef]

24. Wang, H.F.; Li, C.Z. Bäcklund transformation of Frobenius Painlevé equations. Mod. Phys. Lett. B 2018, 32, 1850181. [CrossRef]

25. Wang, H.F.; Zhang, Y.F. Residual Symmetries and Bäcklund Transformations of Strongly Coupled Boussinesq-Burgers System. Symmetry 2019, 11, 1365. [CrossRef]

26. Kompaneets, A.S. The establishment of thermal equilibrium between quanta and electrons. Z. Eksp. Teor. Fiz 1956, 31, 876-885.

27. Ma, S.L.; Wu, Y.M.; Zuo, D.F. The Frobenius-Virasoro algebra and Euler equations-II: Multi-component cases. J. Geom. Phys. 2019, 135, 32-41. [CrossRef]

28. Wang, Z.L.; Zhang, L.H.; Li, C.Z. Lie symmetry analysis to weakly coupled Kaup-Kupershmidt equation with time fractional order. Fractals 2019, 27, 1950052. [CrossRef]

Publisher's Note: MDPI stays neutral with regard to jurisdictional claims in published maps and institutional affiliations.

(C) 2020 by the authors. Licensee MDPI, Basel, Switzerland. This article is an open access article distributed under the terms and conditions of the Creative Commons Attribution (CC BY) license (http:/ / creativecommons.org/licenses/by/4.0/). 